\title{
Optimization Performance Analysis of 1090ES ADS-B Signal Separation Algorithm based on PCA and ICA
}

\author{
Zhaoyue Zhang* \\ College of Air Traffic Management, Civil Aviation University of China, Tianjin, 300300, China
}

\begin{abstract}
In the transmission process of 1090ES ADS-B signal, it is easy to see the relationship between signal overlap and signal interference. The signal noise under interference easily causes the error of ADS-B signal decoding. In this paper, a 1090ES ADS-B signal separation algorithm based on PCA and FastICA is designed to achieve the separation of ADS-B signals and the separation of overlapped signals. Through simulation and verification, the 1090ES ADS-B signal separation algorithm based on PCA and ICA can realize the denoising and separation of ADS-B signal and reduce the computation and speed of separation. The signal reduction degree after separation is relatively high.
\end{abstract}

Keywords: PCA; FastICA; ADS-B; signal separation

(Submitted on January 15, 2018; Revised on February 18, 2018; Accepted on March 29, 2018)

(C) 2018 Totem Publisher, Inc. All rights reserved.

\section{Introduce}

The 1090ES ADS-B (Automatic Dependent Surveillance-Broadcast) data link is an aircraft comprehensive communications and surveillance technology. It is an ICAO-approved data link that can transmit flight status information such as aircraft position information, speed, altitude, etc [1]. It has a wider coverage, a lower signal to noise ratio, and a smaller power difference between signals, making it easier to achieve global air surveillance. However, Langejan [7] pointed out in his research that ADS-B also increases signal interference in the process of increasing transmission range. Therefore, degradation of ADS-B signal interference should be considered in future studies. Petrochilos [3] has applied a new algorithm, named projection algorithm (PA), whose idea is to convert a single problem into a multi-channel problem to solve. Later Liu Kai [9] used an iterative projection algorithm (IPA) to solve this problem, and proposed that the separation algorithm should adapt to high-order overlapping. Piracci [10] et al. designed the ADS-B receiver with signal recognition function to detect and solve the problem of signal overlap, but the receiver's built-in algorithm is quite complex. Leonardi [8] et al. proposed a "low-level" method for signal separation in an ADS-B receiver, however, this method does not solve the problem of signal interference well. In addition, Gong [4] used the extended projection algorithm (EPA) and Principal Component Analysis (PCA) algorithm to separate the ADS-B signal, but applying the EPA algorithm requires preprocessing mixed signals in advance and then separating them one by one. Compared to other algorithms, the independent component analysis (ICA) method has low computational complexity and requires less hardware for the device. The algorithm can separate independent signals from the unknown mixed signal, which is suitable for the original signal separation [12]. In this process, there is no need to calculate the higher-order statistics of the signal and has second-order convergence [13]. The PCA method was proposed by Hotelling [5] in 1933. It can effectively eliminate noise and calculate dimensions. Zhen [2] once applied the PCI-ICA method to the separation of noise signals from microphones in industrial environments and achieved good results. Therefore, after comparing the previous ADS-B signal processing method and PCI-ICA application field, this paper proposes an optimized ADS-B signal separation algorithm based on PCA and ICA algorithms.

\footnotetext{
* Corresponding author.

E-mail address: zy_zhang@cauc.edu.cn
} 


\section{ADS-B signal model}

The 1090ES ADS-B uses Pulse Position Modulation (PPM) coding as shown in Figure 1 [11]. The ADS-B message is composed of 4 identification pulses and 56-bit or 112-bit data blocks.

Each bit in the data block takes 1 and uses rising or falling pulses to represent "0" or "1". The first 8 of the ADS-B message pulse is the header signal, and 4 of these are 0.5 high-level signals.

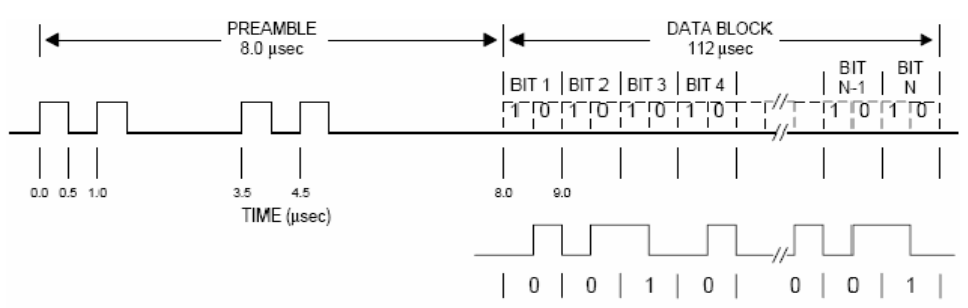

Figure 1. ADS-B message transmission waveform

As shown in Figure 2, this paper uses MATLAB simulation ADS-B signal. The simulation signal is 10Mhz sampling rate with an average of 10 sampling signals for each bit, which can meet the algorithm requirements of ADS-B signal decoding.

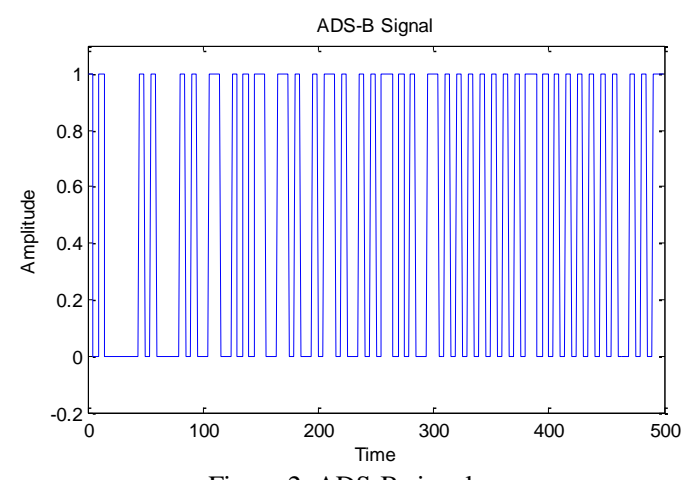

Figure 2. ADS-B signal

ADS-B is a broadcast communication technology. When there are multiple ADS-B transmitting devices in the air, multiple signals will be transmitted simultaneously. Therefore, when multiple signal communications occur at the same time, there may be signal interference or overlap, which interferes with signal decoding. In general, signal interference comes from other ADS-B signals or noise signals during propagation, such as secondary radar pulses or ADS-C interference. Figure 3 shows the overlap of the two ADS-B signals. The signal in the figure cannot be used to determine the signal identification pulse and data block pulse signal. Figure 4 shows the superposition of the ADS-B signal and the noise signal. Although the signal of the superimposed signal and the pulse of the ADS-B source signal are close to each other, the preamble identification pulse cannot provide a power reference for the subsequent decoding.
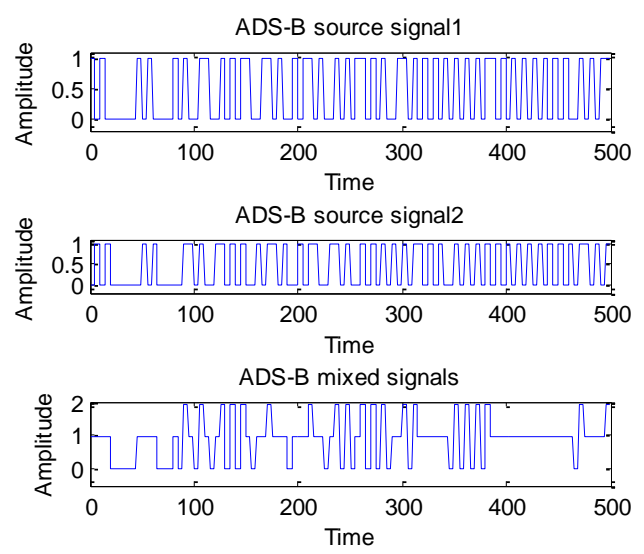

Figure 3. Overlapping of ADS-B signals 

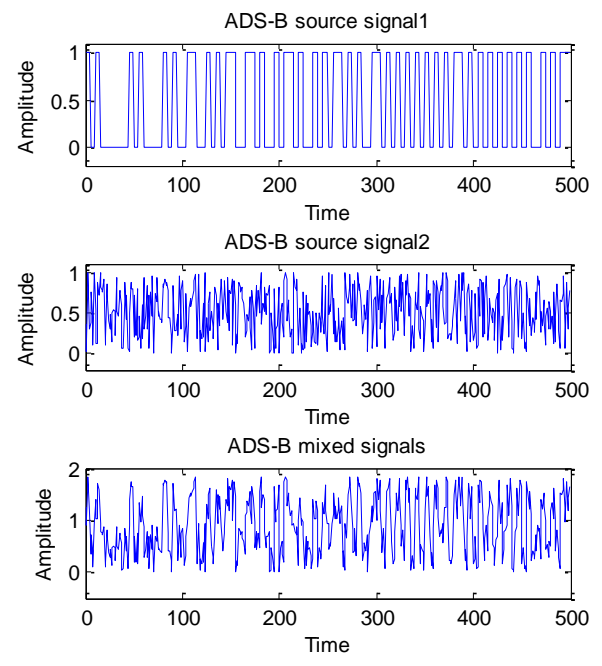

Figure 4. ADS-B and noise signal overlap

In this ADS-B mathematical model, the observation signal $x_{i}(t)$ of ADS-B is defined as the ith observation signal of the ADS-B and $s_{i}(t)$ represents the ith source signal of the ADS-B. Assume that both the ADS-B observation signal and the ADS-B source signal are random signals. $X(t)$ represent the m-dimensional ADS-B observation signal $\left[x_{1}(t), x_{2}(t), x_{3}(t) \cdots x_{m}(t)\right] . \quad S(t)$ indicates the m-dimensional ADS-B source signal $\left[s_{1}(t), s_{2}(t), s_{3}(t) \cdots s_{n}(t)\right] \cdot$ The ith observation signal $x_{i}(t)$ of the ADS-B is overlapped and mixed by $n$ independent ADS-B source signals $s_{i}(t)$. The model is formula (1):

$$
x_{i}(t)=a_{i 1} s_{1}(t)+a_{i 2} s_{2}(t)+\cdots+a_{i n} s_{n}(t), i \text { is an integer }
$$

Where $a_{i j}$ is the element of the mixed matrix $A$, variable $i$ and variable $j$ are integers. There is a noise signal in the actual ADS-B observation signal. If the signal interference $n(t)$ in the propagation process is taken into consideration, the observation signal received by the ADS-B base station can be expressed as formula (2):

$$
X(t)=A S(t)+n(t)
$$

Or express the observation signal $x_{i}(t)$ of the ith ADS-B as formula (3):

$$
x_{i}(t)=\sum_{j=1}^{n} a_{i j} s_{j}(t)+n(t)
$$

\section{Algorithm Description}

The signal separation of ADS-B is divided into two processes: dimensionality reduction and separation. The flow chart is shown in Figure 5. The main process is to separate the data of an independent m-dimensional ADS-B observation signal through PCA algorithm to obtain the dimensionality reduction of the data, and obtain a k-dimensional data vector containing only the characteristic signal. The reduced-dimensional k-dimension signal is used as the input quantity. The FastICA algorithm is used to perform ADS-B signal separation again. The separation matrix is calculated and the ADS-B source signal is separated.

\subsection{PCA algorithm}

The 1090ES ADS-B signal separation algorithm based on PCA and ICA needs to calculate the separation matrix $W$ and separate the source signal $S(t)$ of ADS-B from the observation signal $X(t)$ of ADS-B through the matrix variable $W$. 
This paper uses the PCA algorithm to reduce the dimension of ADS-B signal. Principal component analysis (PCA) is a technique for analyzing data. It simplifies ADS-B source signal data, removes noise and redundancy in ADS-B signals, and reduces the dimensions of the original complex data so that the signal variables after dimensionality reduction represent the original variable's information as much as possible; in addition, they are independent of each other.

To use the PCA algorithm for the ADS-B observation signal, we first need to obtain the observation data matrix of the m-dimensional ADS-B base station, as shown in the following formula (4):

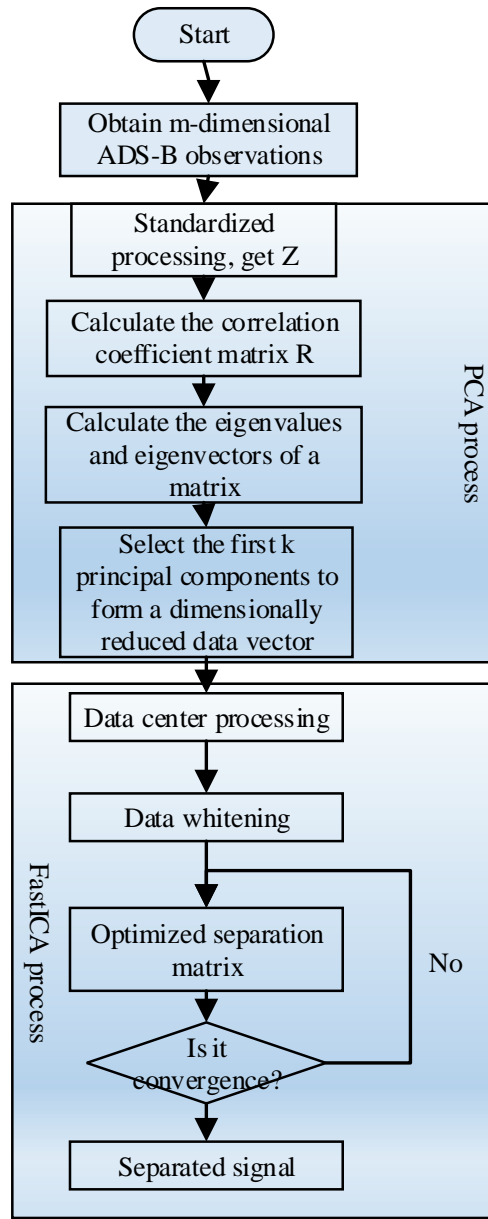

Figure 5. Data processing flowchart

$$
X=\left(\begin{array}{cccc}
x_{11} & x_{12} & \cdots & x_{1 m} \\
x_{21} & x_{22} & \cdots & x_{2 m} \\
\vdots & \vdots & \vdots & \vdots \\
x_{n 1} & x_{n 2} & \cdots & x_{n m}
\end{array}\right)
$$

Standardize the observation data of ADS-B base stations, get a standardized array, as in equation (5):

$$
z_{i j}=\frac{x_{i j}-\bar{x}_{j}}{s_{j}}(i=1,2, \cdots, n ; j=1,2, \cdots, m)
$$

Where $\bar{x}_{j}=\frac{1}{n} \sum_{i=1}^{n} x_{i j}$.

$$
s_{j}^{2}=\frac{1}{n-1} \sum_{i=1}^{n}\left(x_{i j}-\bar{x}_{j}\right)^{2}(j=1,2, \cdots, m)
$$


The correlation coefficient matrix of the ADS-B observation data is calculated, such as formula (6):

$$
R=\left[\begin{array}{cccc}
r_{11} & r_{12} & \cdots & r_{1 p} \\
r_{21} & r_{22} & \cdots & r_{2 p} \\
\vdots & \vdots & \cdots & \vdots \\
r_{p 1} & r_{p 2} & \cdots & r_{p p}
\end{array}\right]=\frac{Z^{T} Z}{n-1}
$$

Where the correlation coefficient of data after standardized processing is defined as formula (7):

$$
r_{i j}=\frac{1}{n-1} \sum_{t=1}^{n} x_{t i} x_{t j}(i, j=1,2, \cdots, m)
$$

Then, the Jacobian method is used to solve the characteristic equation $\left|R-\lambda_{m}\right|=0$ of the sample correlation matrix $\mathrm{R}$ to obtain $\mathrm{m}$ eigenvalues to determine the principal components. The principal component analysis can obtain $\mathrm{m}$ principal components and select the former $k$ principal components according to the cumulative contribution rate of each principal component. The selection of principal component number $k_{k}$ is determined by $\frac{\sum_{j-1}^{k} \lambda_{j}}{\sum_{j=1}^{m} \lambda_{j}} \geq 0.85$, making the utilization rate of ADS-B information more than $85 \%$, and converting the standardized index variables into the main components.

\subsection{ICA Algorithm}

Independent Component Analysis (ICA) is a very effective data analysis tool proposed in recent years. It is mainly used to extract original independent signals from mixed data.

In order to ensure that the ICA model is solvable, the following assumptions and restrictions need to be made [6]:

- The source signal has statistical independence

- The input source signal conforms to a non-Gaussian distribution

- The unknown mixed matrix is full rank, i.e. the number of independent components is equal to the number of observed mixed signals

In the existing ICA algorithm, the fixed-point algorithm (FastICA) is widely used in the signal processing field because of its fast convergence speed and good separation effect. The algorithm is based on the fixed-point recursive algorithm. It is a fast optimization iterative algorithm, making the convergence faster and more robust.

In FastICA algorithm, the ADS-B observation signal $X$ after PCA dimensionality reduction needs to be centralized so that its mean value is 0 ;

Then, the processed ADS-B observation signal data is whitened, $X \rightarrow Z$;

Select the number of components $m$ to be estimated, and set the number of initialization iterations $p \leftarrow 1$;

Select an initial weight vector (random) $W_{p}$, and let $W_{p}=E\left\{Z g\left(W_{p}^{T} Z\right)\right\}-E\left\{g^{\prime}\left(W_{p}^{T} Z\right)\right\} W, g$ as a non-linear function $g(y)=\tanh (y)$

Calculate $W_{p}=W_{p} /\left\|W_{p}\right\|$, where $W_{p}=W_{p}-\sum_{j=1}^{p-1}\left(W_{p}^{T} W_{j}\right) W_{j}$.

\section{Simulation and Analysis}

In order to verify the feasibility of PCA and FastICA in ADS-B signal separation, the separation of three overlapping ADS$\mathrm{B}$ signals and the ADS-B signals under the noise signal are verified. In the simulation experiment, ADS-B signal sampling is 
10Mhz, and the sampling data is 500 samples. The signal received by each ADS-B base station is a random superposition of ADS-B signals, respectively.

\subsection{Triple Signal Separation Algorithm}

In the triple signal separation simulation, Figure 6 shows the ADS-B source signals, which is pulse modulation encoded. Each bit has 10 sampling signals, including 8us header signals and 42us message signals, and the message bits are randomly generated. The ADS-B source signal is superimposed by a $5 \times 3$ random matrix as five-channel observation signals, which is simulated as a base station of five-channel observation signals, as shown in Figure 7. There are problems in the 5-channel observation signals that signals cannot be directly decoded. If the signals are directly separated, the computing resources will be wasted. Therefore, the dimensionality reduction of the signal is first performed by the PCA, and the characteristic signal of the signal is extracted, as shown in Figure 8. The signals after dimension reduction contain only three signals of the characteristic signal, which satisfies the signal separation requirements of the analog source signal. For signals after dimension reduction, FastICA is used to separate the ADS-B signal. The separated signals are shown in Figure 9, and the decoded signal is basically the same as the simulated 3-channel ADS-B signal. Figure 10 shows the principal component contribution rate of the ADS-B signal in the PCA algorithm.
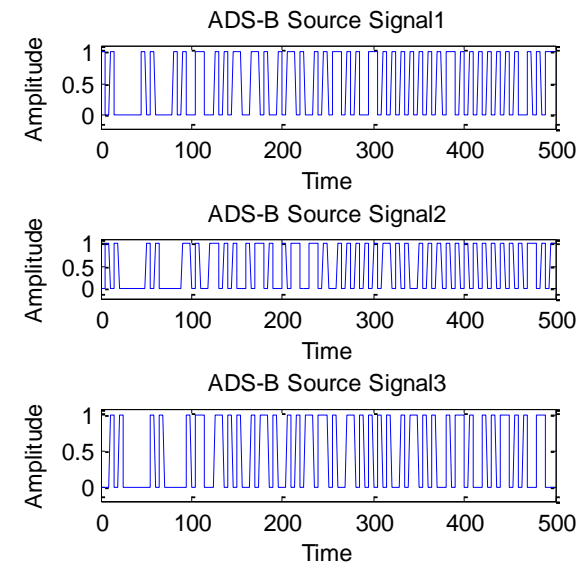

Figure 6. ADS-B source signals
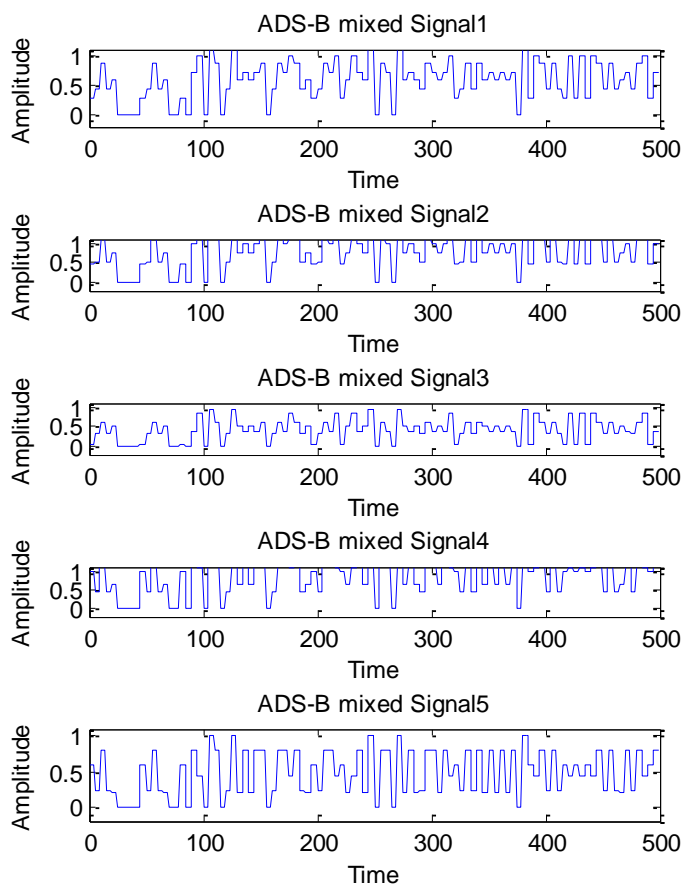

Figure 7. ADS-B observation signals 

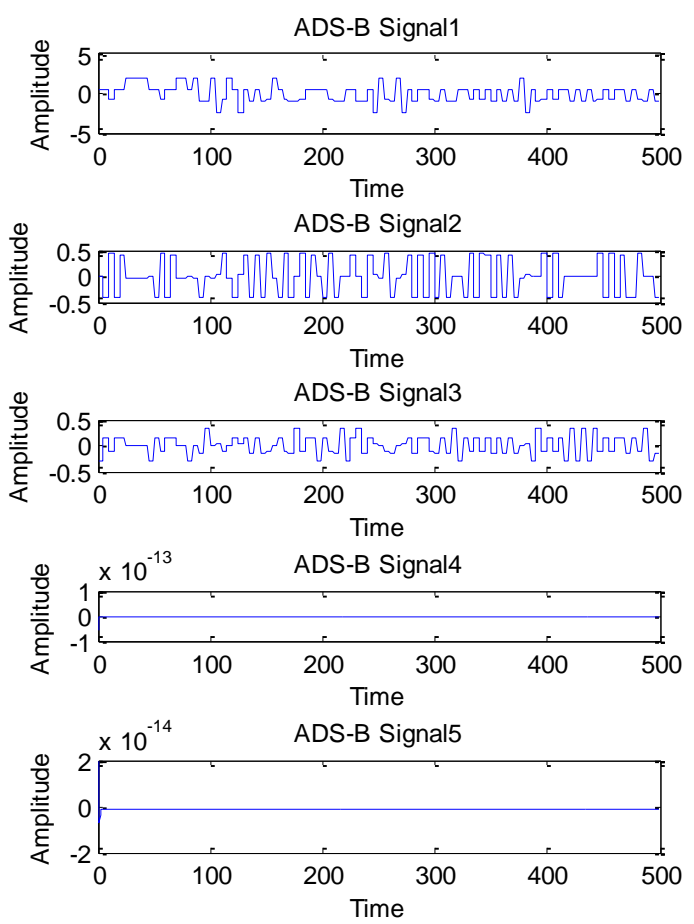

Figure 8. Signals after dimension reduction
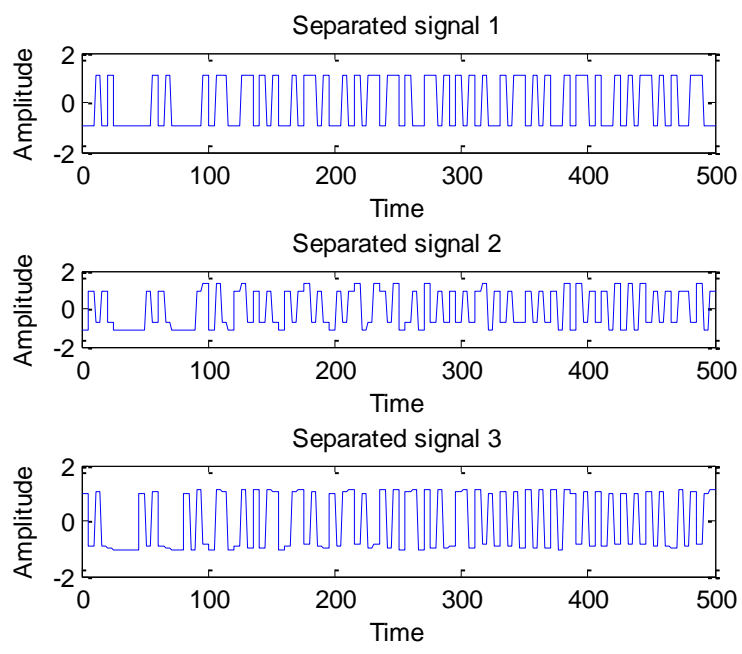

Figure 9. Separated signals

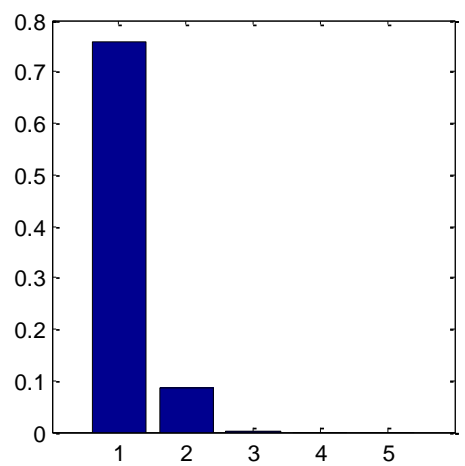

Figure 10. Principal component contribution rate 


\subsection{ADS-B signal separation with noisy signal}

The above verifies the feasibility of PCA and FastICA in ADS-B signal, and will subsequently verify the separation of the ADS-B signal under the noise signal. The random noise and impulse noise that may affect the ADS-B signal are added to the ADS-B source signal, as shown in Figure 11. The first and the second figure in Figure 11 are ADS-B signals added random noise, and the third figure in Figure 11 is added the interference pulse signal and other random noise signals. Such a superimposed source signal will have a great impact on decoding of the ADS-B signal. In this paper, the random matrix is still used to randomly superimpose three-channel signals into five-channel signals as observation signals of ADS-B base station, as shown in Figure 12. PCA is used to extract characteristic signals of the 5-channel signal, the amount of calculation is reduced, and the effect of signal dimension reduction is shown in Figure 13. The extracted characteristic signal is 3-channel, which satisfies the signal processing requirements. The FastICA algorithm is used to separate the ADS-B signal after dimension reduction, as shown in Figure 14.
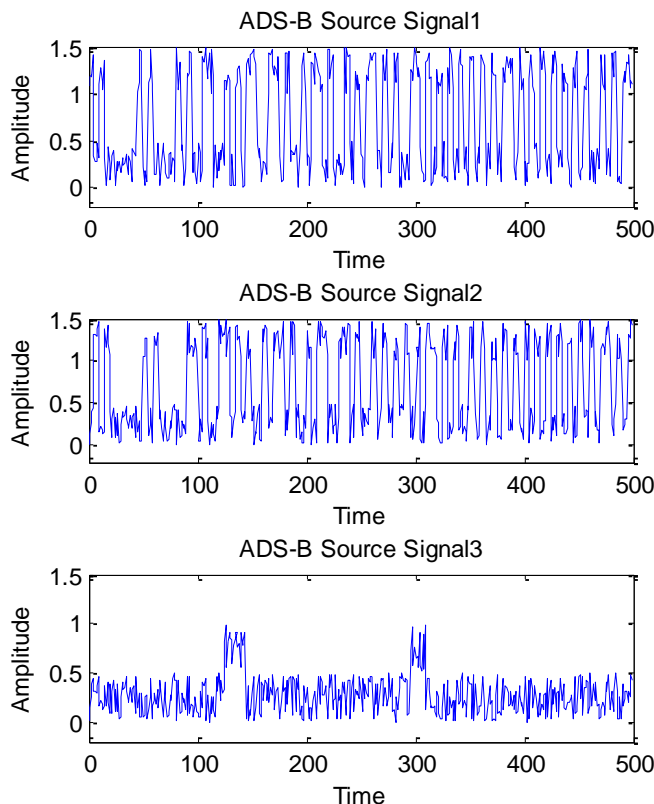

Figure 11. ADS-B source signals under noise
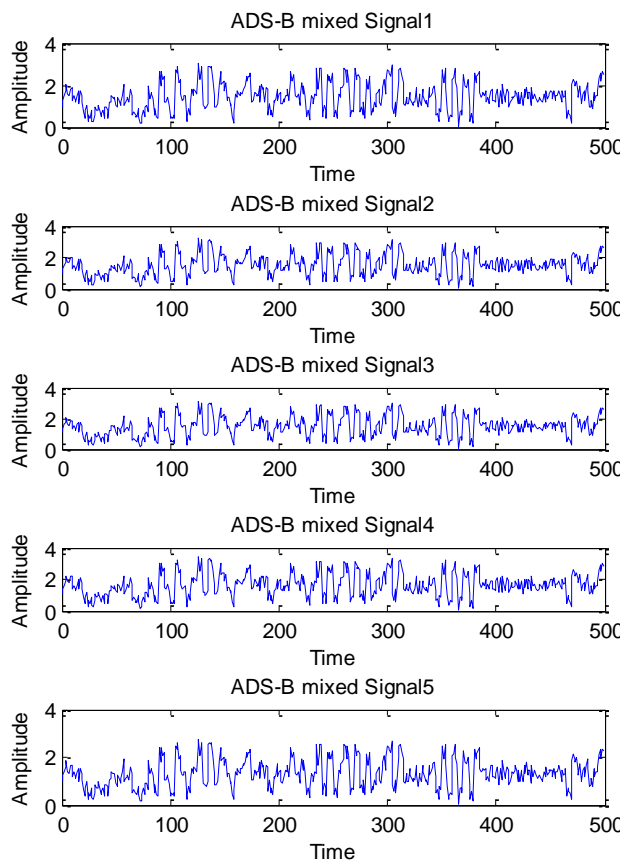

Figure 12. ADS-B observation signals under noise 

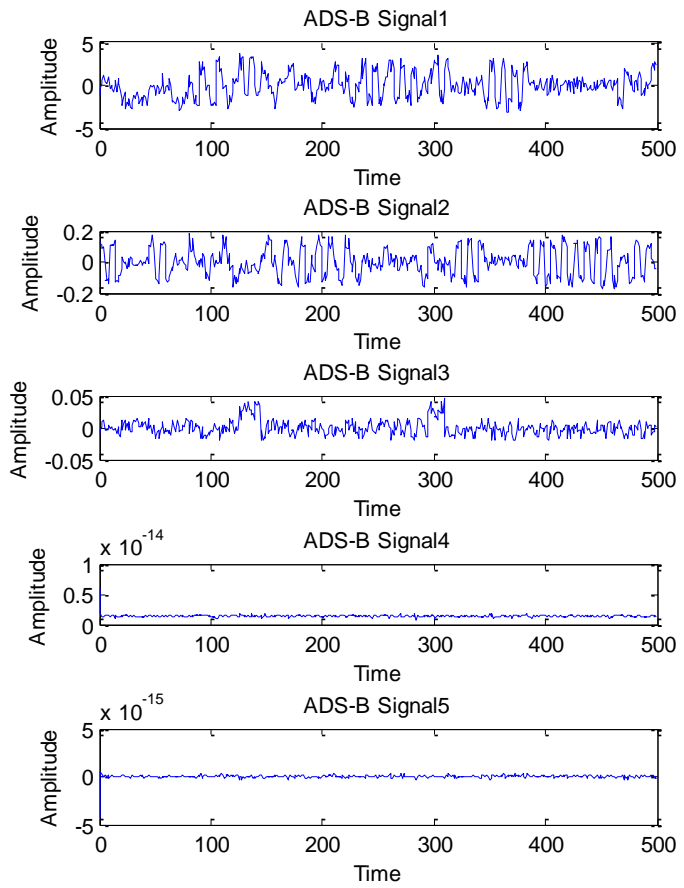

Figure 13. Signals after PCA processing

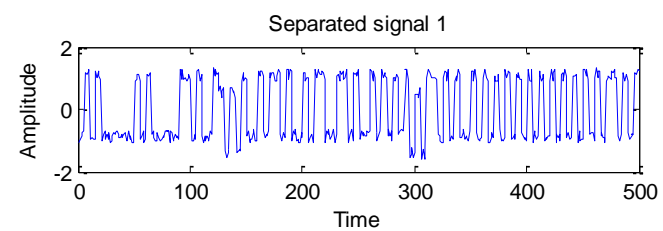

Separated signal 2

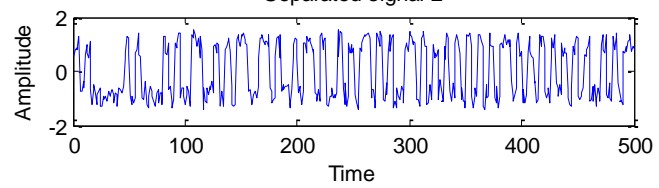

Separated signal 3

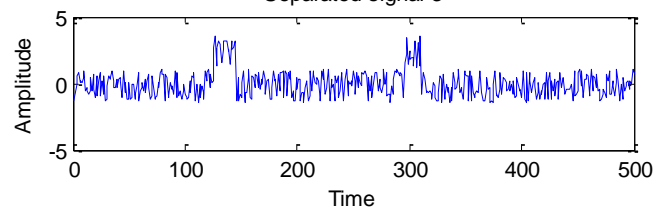

Figure 14. Separated signals

\subsection{Analysis of simulation results}

From the simulation results, the algorithm based on PCA and FastICA can meet the signal dimension reduction and signal separation processing. In triple signal separation simulation experiment, the extracted dimension of the main component of the signals and the separation of the signals can satisfy the signal processing requirements of ADS-B. The use of the PCA algorithm reduces the computation of the multi-dimensional ADS-B observation signals and improves the ADS-B signal processing speed. After adding random noise and interference signal, PCA algorithm is used to effectively filter out some of the interference noise, which makes the signal level stability has improved. The use of FastICA can effectively separate the ADS-B signals and also separate out the interference source pulse signal.

\section{Conclusions}

The 1090ES ADS-B signal separation algorithm based on PCA and FastICA reduces the computational complexity of the signal processing and improves the speed of ADS-B signal separation, making the ADS-B signal under signal overlapping and signal interference still effectively solve the problem of ADS-B signal decoding. Compared with the traditional 
algorithm, this algorithm improves the operation efficiency and guarantees the signal quality at the same time. The disadvantage is that there are multiple solutions in FastICA that meet the assumptions. The signal transformation can meet the decoding requirements and realize the recovery and reconstruction of ADS-B source signals.

\section{References}

1. B. S. Ali, "System specifications for developing an Automatic Dependent Surveillance-Broadcast (ADS-B) monitoring system[J]," International Journal of Critical Infrastructure Protection, 2016, 15:40-46.

2. S. Ao, L. Zhen, Z. Nan, et al, "Blind Source Separation Based on Principal Component Analysis-Independent Component Analysis for Acoustic Signal During Laser Welding Process[C] ," International Conference on Digital Manufacturing \& Automation. IEEE Computer Society, 2010:336-339.

3. G. Galati, N. Petrochilos, E. G. Piracci, "Degarbling Mode S replies received in single channel stations with a digital incremental improvement[J]," Radar Sonar \& Navigation Iet, 2015, 9(6):681-691.

4. F. X. Gong, K. Wang, Y. Q. Ma, “An Algorithm to Separate 1090ES ADS-B Signals[J],” Advanced Materials Research, 2014, 875-877:2158-2163.

5. H. Hotelling, "Analysis of a complex of statistical variables into principal components.[J]," British Journal of Educational Psychology, 1933, 24(6):417-520.

6. A. Hyvarinen, J. Karhunen, "Independent Component Analysis[M]," New York, 2011.

7. T. Langejan, E. Sunil, J. Ellerbroek, et al, "Effect of ADS-B Characteristics on Airborne Conflict Detection and Resolution[C]," Sesar Innovation Days. 2016.

8. M. Leonardi, E. Piracci, G. Galati, "ADS-B vulnerability to low cost jammers: Risk assessment and possible solutions[C]," Tyrrhenian International Workshop on Digital Communications - Enhanced Surveillance of Aircraft and Vehicles. IEEE, 2014:41-46.

9. K. Liu, T. Zhang, Y. Ding, "Blind Signal Separation Algorithm for Space-based ADS-B[C]," International Conference on Mechatronics Engineering and Information Technology. 2016.

10. E. G. Piracci, G. Galati, N. Petrochilos, "1090 ES receiving capacity improvement using ADS-B ground receivers with signals discrimination capability[C],” Esavs 2010 16-18/03/. 2010.

11. RTCA, "DO-260B: Minimum Operational Performance Standards for 1090 MHZ Extended Squitter Automatic Dependent Surveillance-Broadcast (ADS-B) and Traffic Information Services Broadcast (TIS-B) [S]," 2013.

12. H. Zayyani, M. Babaie-Zadeh, C. Jutten, "Source Estimation in Noisy Sparse Component Analysis[C]," International Conference on Digital Signal Processing. IEEE, 2007:219-222.

13. W. Zhou, L. Jia, "A novel fast algorithm for blind signal separation[C]," Intelligent Control and Automation, 2002. Proceedings of the, World Congress on. IEEE, 2002:2007-2010 vol.3. 